of albumin affords. Furthermore, before a synthesis can be successfully attempted, it is necessary to know whether these substances are primary or secondary products formed directly or indirectly by the action of the reagent, and here again the field is still untraversed.

Whilst great similarity exists among the constituent parts of the molecule, there is a wide difference in the proportions of each present. The protamines, which are obtained from the milt (testicles) of fishes, and represent some of the simplest proteids, contain a large proportion of diaminoacids and a small quantity of monoamino-acids; the kindred histones, on the other hand, contain a much smaller proportion of diamino-acids, but the whole group of monoamino-acids. Through a series of gradations we arrive finally at substances like the proteids of silk and elastin, which are exceptionally rich in monoamino-acids.

A comparison of the composition of the individual albumins of food and of the living body leads to the conclusion that in digestion deep-seated changes must occur. Moreover, the view is steadily growing that the albumin molecule forms the basis of the two other important groups of food-stuffs, fats and carbohydrates. The decomposition which certain albumin fragments undergo promises to throw new light on the changes which occur in the organism and on the formation of pathological products.

Granted that the whole series of albumin products were known, their relative arrangement in the molecule would still remain to be discovered. Attempts have been made by using milder reagents to arrest the process of degradation at an earlier stage, and so obtain larger molecular fragments; but great practical difficulties attend the method. Nevertheless, by the labours of Fischer and Bergell a series of no less than four intermediate products between silk and its lowest degradation product have been isolated. First, sericoine; secondly, a substance containing tyrosine; thirdly, one free from tyrosine; and lastly, a compound which probably belongs to the dipeptides mentioned below. This study of partial degradation of the albumin molecule derives increased interest from the behaviour of food albumin in the intestine, which, as Fischer and Abderhalden have shown by their experiments on dogs, probably undergo neither slight nor yet complete decomposition, but partial hydrolysis. It will be an attractive problem to determine how far food albumin may be degraded and yet afford nutriment for the organism.

If the process of decomposition cannot furnish the necessary information about the structure of the albumin molecule, the reverse process of synthesis may effect the desired object. E. Fischer has with wonderful experimental ingenuity and skill successfully followed this path of research. The classical memoirs on the polypeptides have already been referred to in the pages of NATURE. By combining two molecules of amino-acids, the dipeptides, glycyl-glycine, alanyl-alanine, and leucyl-leucine have been obtained, as well as mixed dipeptides, e.g. glycyl-alanine, alanyl-glycine, \&c. By uniting three and more molecules, tri- and tetra-peptides, \&c., are formed. The longest chain of this character is pentaglycine, consisting of a group of five linked glycine molecules.

But, as we have seen, the degradation products of albumin are not all monoamino-acids, but include hydroxyand diamino-compounds, and peptides of these substances have also been prepared. These products show an unmistakable likeness to the natural peptones. They give the usual reactions-the biuret reaction, precipitation by phosphotungstic acid, and hydrolysis by trypsin. A peculiar interest centres round the different behaviour of the peptides towards the pancreatic ferment. Whereas glycyl-l-tyrosine and glycyl-l-leucine are easily hydrolysed, glycyl-glycine and glycyl-alanine are unattacked by the ferment.

These experiments, as Fischer has pointed un, are not only useful in indicating the physiologically important compounds among the numerous synthetic materials obtained by him, but show, by the experience so gained, the possibility of discovering the different kinds of linking which exist among the amino-acids of the albumin molecule.

We may anticipate from these investigations some knowledge of the cause whereby different parts of the molecule resist or retard the action of the ferment.

J. B. C.

\section{ON THE ORIGIN OF EOLITHS.}

FOR some time past the question of the existence of man in different countries during the Tertiary period, based upon flints bearing traces of intentional work, has occupied the lively attention of "prehistorians" in numerous parts of the globe-i
Germany, Russia, Egypt, India, \&c.

According to the eminent Belgian geologist, M. Rutot, who has placed himself at the head of this new movement, we must add to the Palæolithic and Neolithic periods a period more ancient still, which has received the name of Eolithic. This does not comprise any type of instrument chipped into an intentional form, but only natural forms utilised at once. These primitive and rough tools have received the name of eoliths. It is believed that they may be recognised by the presence of secondary work (retouches), that is to say, the removal of small flakes in apparently a systematic manner, in accordance with the needs of the case, or resulting from the wear of the flint by use.

An enormous quantity of eoliths are found in the Quaternary gravels mixed with instruments of determinate and classic forms. In the gravels of the north of France and of Belgium, M. Rutot has described several industries of this kind, the Reutelian, the Mafflian, the Mesvinian, \&c. But such objects are equally met with in beds of far greater antiquity; the chipped stones of the Oligocene of Thenay, of the Miocene of Otta and Aurillac, of the Pliocene of England, \&c., are eoliths; and here the question becomes far more grave, inasmuch as the adepts in the new theories rely on these facts to admit the existence of man or his immediate precursor during the Tertiary period.

For twenty years I have not ceased to combat these theories; first, because it appeared to me to be imprudent to admit the existence of Tertiary man in the absence of all direct, that is to say, in the absence of osteological evidence, and secondly, because I have always been convinced that the eoliths are due to natural causes. I had, indeed, had occasion to meet with them in all the ancient alluvia of torrential character in which flints were present. In Auvergne, and in the Velay, in the course of my explorations in connection with the geological map, I had found them at numerous points in the midst of Oligocene or Miocene beds occupying thousands of square kilometres in extent. I asked myself how experiments could be undertaken to solve the problem of the eoliths, when $M$. Laville, of the École des Mines, brought before M. Cartailhac, correspondent of the institute, Dr. Obermaier, and myself some experiments carried on daily, but unintentionally, in an industrial establishment.

There are in the Commune of Guerville, near Mantes, some works in which cement is made from a mixture of chalk and plastic clay. The chalk, as usual, contains blocks of flint which are rejected by the diggers. Trucks convey the chalk from the quarry to the neighbouring works, and deliver it with a certain quantity of clay into circular vats called délayeurs. These are about 5 metres in diameter and I.40 metres in depth. The water which serves them arrives by pipes, and is discharged through lateral sieves, carrying with it the finest particles of the mixture of chalk and clay. The water is set in motion by a horizontal wheel, above the level of the water, but from its spokes are suspended harrows (herses) of castiron dipping into the water; the speed of rotation of the wheel is about 4 metres at its circumference.

The water is thus driven into a tumultuous movement, which carries away not only the particles of chalk and clay, but also a certain number of flints which have escaped the attention of the workmen, and have been thrown into the vats together with the chalk. These flints are therefore subjected to blows one against the other which during a period of twenty-nine hours must be extremely numerous. When the machinery is stopped, the flints remain at the bottom of the vat, where they are covered by a coating of chalk. They are taken out of the vats to be washed and placed in heaps, as they are useful for making concrete.

Now these bits of flint that while in the vats have 1 Translation of a paper by M. Marcelliu Boule in the Comptss rendus of the Paris Academy of Sciences (June 26).

NO. I 870 , vOL. 72] 
undergone the dynamic action of an artificial whirlpool in all points comparable with the dynamic action of a torrential current of water, present all the characteristics of the ancient river-gravels; it is easy to find among them, after a few minutes' search, all the most characteristic forms of eoliths, such as are given as typical. My colleagues and I have been able to make a collection of flints admirably retouchés, identical with the forms called by M. Rutot hammer-stones, planes, notched flints, \&c. We have also collected flints showing the cone of percussion, which is generally regarded as an infallible mark of intentional fashioning.

\section{THE BRITISH ASSOCIATION.}

Inaugural Address by Prof. G. H. Darwin, M.A., LL.D., PH.D., F.R.S., President of the Association.

PART II. ${ }^{1}$

THus far we have been concerned with the almost inconceivably minute, and I now propose to show that similar conditions prevail on a larger scale.

Many geological problems might well be discussed from my present point of view, yet I shall pass them by, and shall proceed at once to Astronomy, beginning with the smallest cosmical scale of magnitude, and considering afterwards the larger celestial phenomena.

The problems of cosmical evolution are so complicated that it is well to conduct the attack in various ways at the same time. Although the several theories may seem to some extent discordant with one another, yet, as I have already said, we ought not to scruple to carry each to its logical conclusion. We may be confident that in time the false will be eliminated from each theory, and when the true alone remains the reconciliation of apparent disagreements will have become obvious.

The German astronomer Bode long ago propounded a simple empirical law concerning the distances at which the several planets move about the sun. It is true that the planet Neptune, discovered subsequently, was found to be considerably out of the place which would be assigned to it by Bode's law, yet his formula embraces so large a number of cases with accuracy that we are compelled to believe that it axises in some manner from the primitive conditions of the planetary system.

The explanation of the causes which have led to this simple law as to the planetary distances presents an interesting problem, and, although it is still unsolved, we may obtain some insight into its meaning by considering what I have called a working model of ideal simplicity.

Imagine then a sun round which there moves in a circle a single large planet. I will call this planet Jove, because it may be taken as a representative of our largest planet Jupiter. Suppose next that a meteoric stone or small planet is projected in any perfectly arbitrary manner in the same plane in which Jove is moving; then we ask how this third body will move. The conditions imposed may seem simple, yet the problem has so far overtaxed the powers of the mathematician that nothing approaching a general answer to our question has yet been given. We know, however, that under the combined attractions of the sun and Jove the meteoric stone will in general describe an orbit of extraordinary complexity, at one time moving slowly at a great distance from both the sun and Jove, at other times rushing close past one or other of them. As it grazes past Jove or the sun it may often but just escape a catastrophe, but a time will come at length when it runs its chances too fine and comes into actual collision. The individual career of the stone is then ended by absorption, and of course by far the greater chance is that it will find its Nirvana by absorption in the sun.

Next let us suppose that instead of one wandering meteoric stone or minor planet there are hundreds of them, moving initially in all conceivable directions. Since they are all supposed to be very small, their mutual attractions will be insignificant, and they will each move almost as though they were influenced only by the sun and Jove.

1 Delivered at Johannesburg on August 30 . The first part of the Address, delivered at Cape Town on August I5, appeared in NATURE of August I7.
Most of these stones will be absorbed by the sun, and the minority will collide with Jove.

When we inquire how long the career of a stone may be, we find that it depends on the direction and speed with which it is started, and that by proper adjustment the delay of the final catastrophe may be made as long as we please. Thus by making the delay indefinitely long we reach the conception of a meteoric stone which moves so as never to come into collision with either body.

There are, therefore, certain perpetual orbits in which a meteoric stone or minor planet may move for ever without collision. But when such an immortal career has been discovered for our minor planet, it still remains to discover whether the slightest possible departure from the prescribed orbit will become greater and greater and ultimately lead to a collision with the sun or Jove, or whether the body will travel so as to cross and re-cross the exact perpetual orbit, always remaining close to it. If the slightest departure inevitably increases as time goes on, the orbit is unstable ; if, on the other hand, it only leads to a slight waviness in the path described, it is stahle.

We thus arrive at another distinction: there are perpetual orbits, but some, and indeed most, are unstable, and these do not offer an immortal career for a meteoric stone; and there are other perpetual orbits which are stable or persistent. The unstable ones are those which succumb in the struggle for life, and the stable ones are the species adapted to their environment.

If, then, we are given a system of a sun and large planet, together with a swarm of small bodies moving in all sorts of ways, the sun and planet will grow by accretion, gradually sweeping up the dust and rubbish of the system, and there will survive a number of small planets and satellites moving in certain definite paths. The final outcome will be an orderly planetary system in which the various orbits are arranged according to some definite law.

But the problem presented even by a system of such ideal simplicity is still far from having received a complete solution. No general plan for determining perpetual orbits has yet been discovered, and the task of discriminating the stable from the unstable is arduous. But a beginning has been made in the determination of some of the zones surrounding the sun and Jove in which stable orbits are possible, and others in which they are impossible. There is hardly room for doubt that if a complete solution for our solar system were attainable, we should find that the orbits of the existing planets and satellites are numbered amongst the stable perpetual orbits, and should thus obtain a rigorous mechanical explanation of Bode's law concerning the planetary distances.

It is impossible not to be struck by the general similarity between the problem presented by the corpuscles moving in orbits in the atom, and that of the planets and satellites moving in a planetary system. It may not, perhaps, be fanciful to imagine that some general mathematical method devised for solving a problem of cosmical evolution may find another application to miniature atomic systems, and may thus lead onward to vast developments of industrial mechanics. Science, however diverse its aims, is a whole, and men of science do well to impress on the captains of industry that they should not look askance on those industry of investigation which may seem for the moment far beyond any possibility of practical utility.

You will remember that I discussed the question as to whether the atomic communities of corpuscles could be regarded as absolutely eternal, and that I said that the analogy of other moving systems pointed to their ultimate mortality. Now the chief analogy which I had in my mind was that of a planetary system.

The orbits of which I have spoken are only perpetual when the bodies are infinitesimal in mass, and meet with no resistance as they move. Now the infinitesimal body does not exist, and both Lord Kelvin and Poincaré concur in holding that disturbance will ultimately creep in to any system of bodies moving even in so-called stable orbits; and this is so even apart from the resistance offered to the moving bodies by any residual gas there may be scattered through space. The stability is therefore only relative, and a planetary system contains the seeds of its own destruction. But this ultimate fate need not disturb 